INVESTIGACIÓN

Recibido: 16/11/2017 --- Aceptado: 21/05/2019 --- Publicado: 15/12/2019

\title{
ANÁLISIS Y LA REGULACIÓN DEL LOBBY EN LA UNIÓN EUROPEA
}

\section{Analysis and regulation of the lobby in the European Union}

Ana Belén Oliver González¹. Universidad Complutense de Madrid. España. anabeleo@ucm.es

\section{RESUMEN}

Actualmente hay miles de grupos de lobbies registrados en Bruselas. El registro de lobbies en la UE es voluntario, no establece sanciones para aquellos grupos que decidan no hacerlo. Factor determinante en la política de transparencia y ética en el proceso de toma de decisiones. El lobby es una actividad opaca por su propia naturaleza, que aunque necesaria para el ejercicio de la democracia, está mediada por el manejo de información -algunas veces de forma privilegiada- que reducen la maniobrabilidad democrática de actores que de por sí no han sido electos para el cargo que ocupan y son más susceptibles a presiones. El objetivo general de este estudio es analizar la regulación del lobby en la UE. Los objetivos específicos son describir las principales características de los lobbies en las instituciones comunitarias, identificar el marco regulatorio comunitario para el ejercicio de lobbies en dichas instituciones. La regulación del lobby ha sido más una respuesta política a la desafección ciudadana que una medida de coerción institucional. No existe un marco homogéneo ni de evaluación ni de sujeción que no sea susceptible de vulneración por parte del ejército lobista permanente en Bruselas, como tampoco hay un marco único en cada Estado. Se trata en general de mecanismos novedosos para regular un oficio que hasta no emerger como parte de la crisis de 2008 no constituyó un problema; muy por el contrario, sigue siendo visto como parte fundamental.

PALABRAS CLAVE: lobby - regulación lobbies - instituciones comunitaria - registro de transparencia - confianza institucional.

\footnotetext{
1 Ana Belén Oliver González: Doctora en Ciencias Políticas y Relaciones Internacionales, Universidad Complutense. Máster universitario en Comunicación Política y Empresarial, Universidad Camilo José Cela. Grado en Protocolo y Relaciones Institucionales, Universidad Camilo José Cela. anabeleo@ucm.es
} 


\begin{abstract}
There are currently thousands of lobby groups registered in Brussels. The registration of lobbies in the EU is voluntary, it does not establish penalties for those groups that decide not to do so. Determinant factor in the policy of transparency and ethics in the decisionmaking process. The lobby is an opaque activity by its very nature, which although necessary for the exercise of democracy, is mediated by the handling of information sometimes in a privileged way- that reduces the democratic maneuverability of actors who in themselves have not been elected for the position they occupy and are more susceptible to pressure. The general objective of this study is to analyze the regulation of the lobby in the EU. The specific objectives are to describe the main characteristics of lobbies in community institutions, identify the community regulatory framework for the exercise of lobbies in these institutions. Lobby regulation has been more a political response to citizen disaffection than a measure of institutional coercion. There is no homogeneous framework for evaluation or restraint that is not susceptible to violation by the permanent lobbyist army in Brussels, nor is there a single framework in each State. These are, in general, novel mechanisms to regulate a trade that until it did not emerge as part of the 2008 crisis was not a problem; On the contrary, it is still seen as a fundamental part.
\end{abstract}

KEY WORDS: lobby - regulation lobbies - community institutions - transparency registry - institutional trust.

\title{
ANALISES E A REGULAÇÃO DO LOBBY NA UNIÃO EUROPEIA
}

\section{RESUME}

O registro de lobbies na EU é voluntario, não estabelece sanções para aqueles grupos que decidam não o fazer. Fator determinante na política de transparência e ética no processo de tomada de decisões. O lobby é uma atividade opaca por sua própria natureza, que apesar de necessária para o exercício da democracia, esta mediada pelo manejo de informação - algumas vezes de forma privilegiada - que reduzem a manobralidade democrática de atores que por si mesmo não foram eleitos para o cargo que ocupam e são mais susceptíveis a pressões. $O$ objetivo geral deste estudo é analisar a regularização do lobby na EU. Os objetivos específicos são descrever as principais características dos lobbies nas instituições comunitárias, identificar o marco regulatório comunitário para o exercício de lobbies nas citadas instituições. A regularização do lobby foi mais uma resposta política à insatisfação da cidadania que uma medida de coerção institucional. Não existe uma referência homogênea nem de valoração nem de sujeição que não seja susceptível de vulneração por parte do exército lobista permanente em Bruxelas, como tampouco há um marco único em cada Estado. Se trata em geral de novidades de mecanismos para regular um oficio que até não emergir como parte da crise de 2008 não constituiu um problema; muito pelo contrário, segue sendo visto como parte fundamental. 
PALAVRAS CHAVE: lobby - regulamento lobbies - instituições comunitárias, registro de transparência - confiança institucional.

\section{Como citar el artículo:}

Oliver González, A. B. (2019). Análisis y la regulación del lobby en la Unión Europea. [Analysis and regulation of the lobby in the European Union]. Vivat Academia. Revista de Comunicación, (149), 91-108.

doi: http://doi.org/10.15178/va.2019.149.91-108

Recuperado de http://www.vivatacademia.net/index.php/vivat/article/view/1107

\section{INTRODUCCIÓN}

En la actualidad hay miles de grupos de lobbies registrados en Bruselas, de distintos tipos y diversas procedencias, desde grupos empresariales sectoriales, defensores del medioambiente, fundaciones extranjeras, hasta la llamada paradiplomacia de regiones y facciones de entidades territoriales de Estados Miembros.

Este caleidoscopio de intereses y presiones de grupos específicos no es nada nueva, aunque los procesos de transparencia y regulación impulsada por diversos sectores para legalizar el trabajo de facto ejercido por los lobbies sí resulta novedoso, ya que es a partir de 2008 que se iniciará un registro en Bruselas sobre estos grupos y su puesta en práctica se desarrollará a partir de 2011 en los tres principales órganos comunitarios susceptibles de lobby: la Comisión Europea, el Consejo Europeo y el Parlamento Europeo.

La Comisión Europea, en adelante la Comisión, estima que en Bruselas se dan vida más de 2500 lobbies profesionales, con un "ejercito" de cabildeo que puede llegar a más de 20.000 personas; su presencia se destaca a partir de 1992 con la entrada en vigencia del Tratado de Maastricht, aunque sus orígenes estarían de forma informal y residual a mediados de los setenta del siglo XX, que actuaban sin regulaciones y que su trabajo resultaba más opaco al ser el lobby diplomático el que ejercía el rol protagónico en la capital europea.

El registro de lobbies en la UE es de carácter voluntario, es decir, no establece sanciones para aquellos grupos que decidan no hacerlo. De ahí que los principales críticos establezcan parámetros diferentes para calcular un número específico, toda vez que tal registro no llega a los 8000 grupos lobistas apuntados. Este factor es determinante en la política de transparencia y ética en el proceso de toma de decisiones de las instituciones comunitarias.

Por su parte, Cataluña (España), Escocia (Reino Unido) Baja Sajonia (Alemania) o Venecia (Italia), entre otras; son regiones territoriales de sus países con oficinas 
permanentes en la capital europea para presionar por sus intereses domésticos y persuadir en el proceso de toma de decisiones, bien sea en el Parlamento Europeo, en el Consejo Europeo, o en la Comisión Europea, donde ejercen un fuerte lobby hacia proyectos de financiamiento comunitario o frente a políticas sensibles para sus economías, como la PAC (Política Agrícola Común).

Mientras la Comisión lidera la iniciativa comunicacional de Bruselas, y el Parlamento con su sede principal en Estrasburgo asienta todo el arco político europeo -y sus asistentes, oficinas, burócratas y lobistas detrás-, es el Consejo Europeo el que requiere de un trabajo mucho más delicado, atinado del cabildeo profesional, dada su Alta Representación y la definición de la agenda que ocurre en su seno.

Mucho menos mediático aunque de mayor riesgo, el Consejo Europeo es la máxima oficina de representación de la UE, por lo que su presidente está sometido a ingentes presiones de diversos y variados actores (de los propios Estados, de las otras instituciones de la UE, de los intereses inter-agencias de la UE, de los territorios de los Estados, de los grupos empresariales globales, de las organizaciones internacionales, etc.) y dado el caleidoscopio al que sirve, sus decisiones están sujetas a la satisfacción de quienes le componen, es decir, tiene limitaciones fácticas para satisfacer muchos intereses en pugna.

El lobby es una actividad opaca por su propia naturaleza, que aunque necesaria para el ejercicio de la democracia y los derechos, está mediada por el manejo de información -algunas veces de forma privilegiada- que reducen la maniobrabilidad democrática de actores que de por sí no han sido electos para el cargo que ocupan, es decir, son más susceptibles a las presiones de quienes fungieron como sus electores. Teniendo en claridad ello, la UE viene desarrollando importantes avances para reducir tales presiones en sus órganos comunitarios.

\section{OBJETIVOS}

El objetivo general de este estudio es analizar la regulación del lobby en las instituciones de la UE.

Los objetivos específicos son describir las principales características de los lobbies en las instituciones comunitarias, definiendo el marco regulatorio comunitario para el ejercicio de lobbies en dichas instituciones.

\section{METODOLOGÍA}

El desarrollo de la investigación se aborda siguiendo las premisas del método comparativo para investigaciones, mediante el cual se realiza una contrastación entre las 
acciones comunicativas, las políticas de regulación y transparencia, y las políticas persuasivas que influyen en las instituciones comunitarias y estados miembros por parte de los lobbies, observando sus características y la normativa aplicable en el seno de estas instituciones de la UE.

El estudio se ha abordado desde la combinación distintas perspectivas: la normativa y la jurídica. Si bien es cierto que el grado de intensidad con que se ha trabajado cada una de ellas ha sido distinto, se ha procurado que ninguna quedara al margen de este estudio.

Desde un punto de vista normativo, se ha partido de los Tratados de la Unión Europea actualmente en vigor, y en particular el Tratado de la Unión Europea (TUE), el Tratado de Funcionamiento de la Unión Europea (TFUE) y el Tratado de Lisboa. Desde el punto de vista jurídico, se ha trabajado con las leyes de los estados miembros de la UE. El estudio de estas normas y leyes ha ayudado a definir el presente estudio.

Trabajar con la bibliografía existente ha resultado ya más complejo por la necesidad de seleccionar la que tratase de manera específica la noción de los lobbies y el lobbing en la UE. Cabe decir que la literatura sobre el tema es notablemente amplia, pero en la mayor parte de ocasiones nos encontramos ante estudios de conjunto que salvan con unas pocas líneas o páginas, y sin profundizar, el objeto de este estudio; análisis de la resgulación del lobby en las instituciones europeas. Aun así, ha resultado especialmente útil, y en cierto modo se ha seguido, la obra de F. Morata (1995), “Influir para decidir: la incidencia de los lobbies en la UE"; E. Alonso (1995), "El lobby en la UE: Manual del buen uso en Bruselas"; P. Bouwen (2002),"Corporate Lobbying in the European Union: The Logic of Access"; J. Xifra (2005), "Los think tank y advocacy tank como actores de la comunicación política"; A. Castillo (2009), "La comunicación de los lobbies en Internet. El ciberactivismo de los Think Tanks"; R. Calduch (1995) "Relaciones Internacionales" y (2011) "Apuntes de la Unión Europea"; R. Correa (2010), "Comunicación: lobby y asuntos públicos"; A. Peña (2011), "Relaciones públicas y lobby. Gestión para la transparencia"; todos ellos citados a lo largo del estudio. Huelga decir que las aportaciones de esta literatura han sido complementadas por otras obras y artículos especializados extraídos de publicaciones, que se han podido consultar en distintas páginas webs, cuyos enlaces se encuentran en al final del estudio, en fuentes utilizadas.

La técnica de investigación es cualitativa en tanto que explica este proceso a partir de la definición, caracterización y regulaciones de los lobbies en las instituciones comunitarias. Se busca con esta técnica una comprensión amplia de la regulación de los lobbies en las instituciones comunitarias a partir de sus estructuras de funcionamiento y procedimientos normativos dichas instituciones en la ciudad de Bruselas, Bélgica. 


\section{DISCUSIÓN}

\subsection{La confianza institucional como legitimadora del lobbying}

La confianza política ubica en las instituciones y las normas de convivencia, el vértice principal de la materialización efectiva de tal confianza. La arquitectura institucional es la base para la maximización de tal proceso, por lo que la confianza a priori debe producir confianza post-facto, un mecanismo que maximiza los potenciales beneficios derivados de la efectividad de la conducta del conjunto de las intituciones.

La confianza institucional supone una evaluación positiva de los atributos más relevantes que hacen a cada institución digna de confianza, como la credibilidad, la justicia, la competencia, la transparencia. Esta confianza parte de la idea base que ésta actuará de forma igualitaria, justa y correcta en apego al marco jurídico vinculante.

Los agentes lobistas son depositarios y al mismo tiempo legitimadores de la confianza política en la institucionalidad democrática. La rectitud, probidad y transparencia entre éstos y los actores políticos son requisito sine cuan non para la garantía del sistema y de forma específica, para legitimar socialmente el ejercicio del cabildeo profesional como parte del sistema.

\subsection{Rendición de cuentas y lobbies en las instituciones comunitarias}

La rendición de cuentas supone que la ley aplica sanciones a los representantes y funcionarios públicos que incurren en omisiones, malas prácticas y abusos del poder público. Por otro lado, significa que los responsables de las instituciones comunitarias tienen que actuar con reciprocidad y atender eficientemente las demandas públicas de la ciudadanía y de los sectores varios representados en Bruselas. Así, las demandas tienen que ser evaluadas en base a información, opiniones, datos y argumentos especializados que sirvan para tomar decisiones racionales en pos del interés general de la población.

Las instituciones legislativas comunitarias revisten especial relevancia para el lobbying, ya que de su organización y de las reglas internas de procedimientos e introducción de leyes y acuerdos se ejercerán las relaciones externas con individuos, grupos de interés, y los lobbies.

Procurando tomar las decisiones más racionales favorables a los intereses generales de los ciudadanos, los legisladores enmarcan sus decisiones en la pluralidad y contraposición de intereses, requiriendo la asistencia técnica de especialistas, agentes profesionales con acceso a sectores específicos ajenos al funcionario público, es decir, requiere la participación de los lobbistas. 
De esta manera, la regulación del lobbying está relacionada con la calidad y profundidad de la democracia. La interacción institucional entre el Estado y los agentesactores de la sociedad civil son en definitiva los mecanismos para evitar la ausencia de ética, opacidad y potencial corrupción de los lobistas en su ejercicio profesional.

\subsection{Regulación de los lobbies en la Unión Europea}

Desde la creación de la CECA -predecesora de la CEE- se han dado lugar en Bruselas la presencia de lobbies tendientes a influir sobre las decisiones de ésta de manera informal. Observa Francesc Morata que esto fue recibido con beneplácito por los líderes políticos europeístas al considerarlos una forma de facilitar el procesos de adherencias del conjunto de instituciones nacionales en el marco europeo.

Los «padres fundadores» y los teóricos neofuncionalistas, como Haas y Lindberg, atribuían una importancia crucial al desarrollo de relaciones estables entre las élites económicas y sociales de los Estados miembros y la alta autoridad de la CECA (predecesora de la Comisión Europea) con objeto de presionar sobre los gobiernos y crear las lealtades necesarias para profundizar en el proceso de integración hasta hacerlo irreversible. (Morata, 1995, p.131)

Será en el marco del papel de los actores no estatales en el proceso de integración europea y los intereses en torno al modelo de interés comunitario que se crearán las bases necesarias para los debates, especialmente en la llamada Acta Única Europea de 1983, cuando los lobbies sean objeto especial de atención ante la evidente pero no regulada influencia que tenían en las legislaciones y directrices que salían de la Comisión. Afirma Jordi Xifra que "las instituciones europeas fueron desde sus inicios sometidas a presión por parte de grupos industriales, de servicios, despachos de abogados que anhelaban que una determinada e interesada perspectiva apareciera en las normas comunitarias". (Xifra, 2000, p. 175)

Los motivos para ejercer una acción de «lobbying» en Bruselas son innumerables. Una razón básica reside en el hecho de que están en juego ganancias o pérdidas evidentes en términos de regulaciones, financiación o influencia. Al mismo tiempo, la complejidad misma del sistema decisorio, más fragmentado organizativamente, menos integrado jerárquicamente y más competitivo que los sistemas nacionales, ofrece una accesibilidad mucho mayor a los grupos organizados. (Morata, 1995, p. 130)

Las instituciones de la UE en la actualidad han coordinado acciones en relación a los grupos de presión como los lobbies a través de dos vías: por medio de la transparencia informativa en torno a las relaciones de la UE con diversos grupos; y por otro lado, a través de un Código de buenas prácticas de carácter voluntario para que los lobbies guíen su conducta a través de éste. Todo ello sustentado en el marco del derecho comunitario. 
El Tratado de Lisboa de 2009 establece que "las instituciones darán a los ciudadanos y a las asociaciones representativas, por los cauces apropiados, la posibilidad de expresar e intercambiar públicamente sus opiniones en todos los ámbitos de actuación de la Unión" siendo que las propias instituciones comunitarias "mantendrán un diálogo abierto, transparente y regular con las asociaciones representativas y la sociedad civil" (art. 2). Continua en su art. 136, b, diciendo que la UE “reconocerá y promoverá el papel de los interlocutores sociales en su ámbito", por lo que facilitará el diálogo entre ellos, dentro del respeto de su autonomía. (Tratado de Lisboa, 2009).

Sin embargo, el trabajo sobre la relación con actores e intereses de la sociedad civil europea viene siendo desarrollado desde antes. En el año 2006 la Comisión Europea publica el Libro Verde de la Trasparencia Europea, tras un trabajo meticulosamente elaborado por el comisario europeo de Administración, Auditoría y Lucha antifraude, Siim Kallas entre 2004 y 2005. En el Libro Verde, la propia Comisión define al lobbying como,

una parte legítima del sistema democrático, independientemente de que esta actividad sea llevada a cabo por ciudadanos individuales, empresas, organizaciones de la sociedad civil así como por otros grupos de intereses o, incluso, firmas que trabajan en nombre de terceras personas, como responsables de relaciones institucionales, «think-tanks» u abogados. (Europe Union, 2010).

Para la Comisión, las organizaciones de la sociedad civil desempeñan un papel de enlace entre los ciudadanos y las instituciones europeas al favorecer el diálogo político y la participación activa de los ciudadanos en la realización de los objetivos de la UE, por lo que su rol es fundamental para legitimar el quehacer institucional del bloque. Ahora bien, la Comisión Europea clasifica a los grupos de interés en organizaciones sin fines de lucro (asociaciones, federaciones europeas, nacionales e internacionales) y organizaciones con fines de lucro (asesores jurídicos, empresas públicas y privadas y consultores). "Las primeras son, en gran medida, aunque no exclusivamente, organizaciones profesionales. Las segundas son personas que suelen actuar por cuenta de terceros para definir y defender los intereses de éstos". (Álvarez y De Montalvo, 2014, p. 370).

\subsection{Revisión de la literatura sobre lobbies}

La literatura sobre el lobby, el lobbying y su impronta en los sistemas políticos está sujeta a la revisión histórica de los parlamentos anglosajones, con especial deferencia en el caso de Reino Unido y EEUU como fuentes originarias del actual ejercicio profesional del cabildeo.

Se trata de estudios empíricos, contextualizados sobre temáticas de interés sociopolítica y por tanto constreñidos en espacio y tiempo a las dinámicas previstas en 
ello. El foco del análisis actual sin embargo está enfocado en las dimensiones de la democracia y la participación ciudadana en ella, relativas a los mecanismos de transparencia, rendición de cuentas y acceso a la información pública, mecanismos que han cuestionado algunas de las acciones ejercidas por grupos de presión dentro de la sociedad civil.

Dada la naturaleza de los lobbies, su vinculación literaria viene como componente añadido de las causas, las consecuencias y/o el impacto que producen en la dinámica internacional, regional y local, especialmente vinculados a los procesos regulatorios, como el caso que nos ocupa en torno a las instituciones comunitarias.

\subsection{Registro de lobbies en la UE}

En el verano de 2011 se produce un acuerdo entre el Parlamento Europeo y la Comisión Europea para el establecimiento de un "Registro de transparencia para las organizaciones y las personas que trabajan por cuenta propia que participan en la elaboración y aplicación de las políticas de la Unión Europea", cuyo ámbito de aplicación será registrar todas las actividades que se realicen "con objeto de influir directa o indirectamente en los procesos de elaboración o aplicación de las políticas y de toma de decisiones de las instituciones de la Unión, independientemente del canal o medio de comunicación utilizado", señala el Acuerdo. (Join Transparency Register Secretariat, 2012).

En este mismo sentido el Parlamento Europeo aprobó en el año 2012 un "Código de conducta de los diputados al Parlamento Europeo en materia de intereses económicos y conflictos de intereses", donde sus parlamentarios deben dejar constancia respecto a los ingresos provenientes de las actividades remuneradas por fuera de la Cámara, en línea con lo dispuesto en 2011 y siguiendo las políticas iniciadas de 1996.

Este registro de 2011 entre el Parlamento y la Comisión es voluntario, fundamentado en la transparencia y acercamiento con el ciudadano que propicia la UE, legitimando y reforzando la ética en el proceso de toma de decisiones al tiempo que refuerza (o intenta) la información disponible en torno a los intereses que se suceden alrededor de las instituciones comunitarias.

Encierra un código de conducta o buenas prácticas que la UE observa necesario para legitimar el trabajo de estos grupos, quienes a juicio de la Comisión son legítimos representantes de la sociedad civil e intereses sectoriales de los diversos gremios y sectores en la UE. En este registro se señala que lo los grupos inscritos deberán:

- Indicarán siempre su nombre y la entidad o entidades que representan o para las cuales trabajan;

- declararán los intereses, objetivos o fines que persiguen $\mathrm{y}$, en su caso, especificarán los clientes o los miembros a los que representan; 
- no obtendrán ni tratarán de obtener información o decisiones de forma deshonesta o recurriendo a una presión abusiva o a un comportamiento inadecuado;

- no darán a entender, en su trato con terceros, que tienen una relación formal con la Unión Europea o cualquiera de sus instituciones, ni falsearán sus datos a efectos de inscripción en el Registro de forma que puedan inducir a error a terceros o a los funcionarios y otros agentes de las instituciones de la Unión Europea. (Registro de Trasparencia, 2016).

Este registro cuenta en 2016 con 9135 organizaciones que de forma voluntaria de han inscrito y decidido actuar conforme el código de conducta estipulado por la legislación comunitaria. Sólo cinco años han pasado desde su aplicación, y su crecimiento ha sido continuo y sucesivo.

Al finalizar el año 2012, en plena crisis financiera global, y de forma especial, ante la crisis de deuda de los países del sur de Europa (Grecia, Portugal y España, más Irlanda), el Registro público contaba con 5.362 organizaciones, clasificadas en:

- Consultorías profesionales, bufetes de abogados y consultores que trabajan por cuenta propia 586;

- Grupos de presión dentro de las empresas y agrupaciones profesionales y comerciales 2.475;

- Organizaciones no gubernamentales 1.692;

- Grupos de reflexión, instituciones académicas y de investigación 408;

- Organizaciones que representan a las iglesias y las comunidades religiosas 29;

- Organizaciones que representan a autoridades locales, regionales y municipales, otros organismos públicos o mixtos, etc. 271.

Los ciudadanos pueden y deben esperar que el proceso de toma de decisiones de la UE sea lo más transparente y abierto posible. Cuanto mayor es la apertura, más fácil resulta garantizar una representación equilibrada y evitar presiones abusivas y el acceso ilegítimo o privilegiado tanto a la información como a los responsables de la toma de decisiones. La transparencia es, a su vez, un elemento clave para fomentar la participación activa de los ciudadanos europeos en la vida democrática de la UE.

El Registro de Transparencia se ha creado para dar respuesta a preguntas básicas como qué intereses se persiguen, quién los defiende y con qué presupuesto. El sistema está gestionado conjuntamente por el Parlamento Europeo y la Comisión Europea. (Registro de Transparencia, 2016).

\subsubsection{Informe 2012 del Registro de Transparencia}

La Secretaría conjunta del Parlamento y la Comisión elaboraron el primer informe anual del Registro de Transparencia Pública de la UE en donde daban cuenta de las 
poco más de 5400 organizaciones que se inscribieron en ese primer año de funcionamiento.

Señalan que de conformidad con el artículo 11 del TUE las instituciones de la UE están abiertas a la contribución de la ciudadanía, las asociaciones representativas y la sociedad civil, guiadas por el esquema de democracia participativa que propugnan sus acciones.

Señalan que el Registro de Transparencia es una especie de "ventanilla única" para que los ciudadanos ejerzan su derecho a estar informados, conociendo las influencias que se produzcan en el proceso de toma de decisiones en los distintos niveles comunitarios.

A través de este proceso de registro simplificado, las entidades registradas se comprometen a respetar un código de conducta que cubre el cumplimiento de los requisitos legales y éticos principios. Desde su origen, una invitación abierta se ha extendido al Consejo a sumarse a este esquema y para todos los demás órganos de la Unión que lo utilizan como un instrumento de referencia. Las entidades inscritas proporcionan la información más esencial sobre sí mismos: la legislación comunitaria realiza un esfuerzo para estimar la movilización financiera que implica esta presión, desde los costos de las oficinas, las personas que participan, de miembros o clientes, así como la cantidad de fondos de la UE que han recibido. (Informe Anual, 2012, p. 4).

Durante ese primer año, la Secretaría confirmaba un modesto avance en los objetivos propuestos, especialmente por las coordinaciones técnicas establecidas entre el Parlamento y la Comisión para la puesta en marcha de la plataforma electrónica y en tiempo real del total de registro combinado que ambas instituciones llevaban de forma separada. De forma tal que, el Informe 2012 señala que los objetivos conseguidos para ese año fueron:

1. Mudanza de las organizaciones registradas en el Parlamento y la Comisión a una misma lista.

2. Lanzamiento y puesta a punto de la plataforma tecnológica para la inscripción de grupos.

3. Desarrollo del sistema de acreditaciones de grupos en el Parlamento y accesos al edificio.

4. Proveer y completar los lineamientos para usuarios sobre la implementación del Anexo II, y las necesidades de retroalimentación con los mismos.

5. Promover el dialogo con el Consejo para su futura participación en el procedimiento compartido del registro.

6. Evaluar y desarrollar metodologías para asegurar la transparencia de las declaraciones registradas por los grupos participantes. $(2012$, p. 8). 
Finalmente, afirmaba la Junta del Registro de Transparencia que la plataforma puesta en práctica funcionaba bien al concluir el primer año, donde las instituciones y órganos de la UE se han ido acoplando al funcionamiento del sistema, lo que hacía que las expectativas aumentaran y los objetivos y metas a fijarse estuvieran alineados con ello. Algunos de éstos son: mejorar el contenido, las normas de procedimiento, las solicitudes de registros; incrementar la comunicación externa para que continúe creciendo las organizaciones inscritas; colocar a disposición del personal laboral del Parlamento y la Comisión las directrices del Registro a través de programas de formación con posibilidad de extensión al conjunto de toda la UE. Señala a manera concluyente: "El esquema sólo alcanzará su plena eficacia si los Miembros y el personal del instituciones comparten la responsabilidad de promover su uso en sus contactos e interacción con organizaciones de terceros". (2012, p.3).

Señala la Junta en su Informe anual de 2012 del conjunto de las instituciones de la UE para que la transparencia y control del trabajo y del activismo de los grupos de presión y de los lobbies este en concordancia con los presupuestos sobre ética y rendición de cuentas de los funcionarios que integran le integran.

Una de las claves explicativas del activismo de los lobbies a nivel europeo reside en el carácter híbrido del sistema comunitario. Sin tratarse de un Estado en sentido estricto, dispone, no obstante, de una de las características fundamentales de todo Estado: la autoridad necesaria para realizar asignaciones imperativas a la sociedad bajo forma de políticas regulativas, distributivas y redistributivas (Mazey y Richardson, 1994). Dotada de un presupuesto apreciable, la UE adopta decisiones de obligado cumplimiento tanto por las Administraciones nacionales como por las empresas y los particulares de los quince Estados miembros. (Morata, 1995, p. 130).

Los resultados parecen indicar que el camino por recorrer todavía es muy largo, especialmente cuando organizaciones como Transparencia Internacional colocan en un puesto muy mediocre la eficiencia de la UE en particular y de todos los países que le componen en general, en materia de transparencia y regulación de los grupos de presión y lobbies que hacen vida en ellos. 
Tabla 1. Garantías y salvaguardas de la UE frente al Lobby.

\begin{tabular}{|c|c|c|c|c|c|}
\hline \multicolumn{6}{|c|}{$\begin{array}{l}\text { ¿Son suficientemente fuertes las salvaguardias contra la influencia indebida y las reglas para } \\
\text { promover el lobby ético en los sistemas políticos de la Unión Europea? } \\
\text { Escala O-100, donde } 0 \text { es la regulación más débil y } 100 \text { la más fuerte. } \\
\text { La puntuación global es la media sin ponderar de los resultados en las tres categorias: } \\
\text { transparencia, integridad e igualdad de acceso. } \\
\text { Los resultados se presentan en orden descendiente, empezando desde arriba por el } \\
\text { país/institución que ha obtenido la más alta puntuación. }\end{array}$} \\
\hline País/Institución & $\begin{array}{l}\text { Puntuación } \\
\text { global de la } \\
\text { regulación } \\
\text { del lobby } \\
\%\end{array}$ & Transparencia & Integridad & $\begin{array}{l}\text { lgualdad } \\
\text { de } \\
\text { acceso }\end{array}$ & Nivel \\
\hline No hay & $70-100$ & $70-100$ & $70-100$ & $70-100$ & $\begin{array}{l}\text { Excelente } \\
(+70)\end{array}$ \\
\hline Eslovenia & 55 & 58 & 58 & 48 & $\begin{array}{l}\text { Satisfactorio } \\
(51-70)\end{array}$ \\
\hline Comisión Europea & 53 & 48 & 49 & 63 & \\
\hline Lituania & 50 & 48 & 40 & 62 & $\begin{array}{l}\text { Insuficiente } \\
(41-50)\end{array}$ \\
\hline Reino Unido & 44 & 34 & 51 & 46 & \\
\hline Austria & 40 & 34 & 42 & 43 & $\begin{array}{l}\text { Muy } \\
\text { Insuficiente } \\
(<40)\end{array}$ \\
\hline Letonia & 39 & 28 & 43 & 48 & \\
\hline irlanda & 39 & 48 & 38 & 31 & \\
\hline Parlamento Europeo & 37 & 45 & 46 & 21 & \\
\hline Paises Bajos & 34 & 25 & 38 & 39 & \\
\hline Polonia & 33 & 29 & 26 & 43 & \\
\hline República Checa & 29 & 19 & 38 & 30 & \\
\hline Estonia & 29 & 24 & 27 & 35 & \\
\hline Francia & 27 & 24 & 30 & 27 & \\
\hline Eslovaquia & 26 & 21 & 23 & 34 & \\
\hline Bulgaria & 25 & 13 & 25 & 38 & \\
\hline Alemania & 23 & 13 & 25 & 30 & \\
\hline Portugal & 23 & 13 & 19 & 37 & \\
\hline España & 21 & 10 & 35 & 17 & \\
\hline Italia & 20 & 11 & 27 & 22 & \\
\hline $\begin{array}{l}\text { Consejo de la Unión } \\
\text { Europea }\end{array}$ & 19 & 17 & 29 & 13 & \\
\hline Hungria & 14 & 8 & 17 & 18 & \\
\hline Chipre & 14 & 7 & 21 & 13 & \\
\hline tia reqional & 31 & 26 & & & \\
\hline
\end{tabular}

Fuente: International Transparency (2015) Lobbying in Europe, en línea.

De conformidad con esta tabla, Eslovenia, y la Comisión Europea, obtienen un aprobado en la investigación de la organización Transparencia Internacional (una puntuación de más del $50 \%$ en el modelo de medición establecido). A pesar de esto 
último, el informe señala algunos vacíos legales, reducidos marcos regulatorios y tardanza en la aplicación de algunas normativas y procedimientos

Lamentable el antepenúltimo lugar ocupado por el Consejo Europeo, quien de acuerdo a Transparencia Internacional tiene una gran capacidad decisoria pero un pobre rendimiento en regulación de los grupos de presión y lobbistas de su entorno. Esta ONG le otorga $19 \%$, casi la mitad de la media regional del $31 \%$.

A sí mismo, los países altamente endeudados y que requirieron asistencia financiera internacional y europea por la crisis de recesión global 2008/2012 ocupan los peores lugares en ese índice (Chipre, España, Italia y Portugal).

\section{CONCLUSIONES}

La UE dispone de recursos y competencias suficientes para atraer la atención de los grupos organizados. Sus decisiones son relevantes en muchos sentidos para los intereses económicos, sociales y territoriales. Pero su administración y procedimientos técnicos le han hecho una organización fragmentada, atomizada entre comités, grupos, agencias y redes; por lo que diferentes grupos disponen de mayores oportunidades de acceso a los centros clave, y en particular a la Comisión, a lo largo del proceso decisorio e incluso durante la fase de implementación de las políticas y resoluciones que se adoptan.

La regulación del lobby ha sido más una respuesta política a la desafección ciudadana que una medida de coerción institucional. La (re)legitimación de los sistemas políticos a través de un conjunto de reformas legales sobre transparencia, rendición de cuentas y acceso a la información han sido pilares básicos de cambios políticos sucedidos en las instituciones comunitarias.

En este ámbito se aprecia un cambio de tendencia respecto del tratamiento jurídico de los lobbies, de manera que frente al silencio que mantenían la mayoría de los sistemas jurídicos sobre dicho fenómeno, con la excepción prácticamente de Alemania, hemos pasado a una situación en la que ya un elevado número de Estados han introducido o han iniciado la regulación de la actividad desarrolla por tales grupos.

La mayoría de estas regulaciones responden a un modelo poco exigente en el que el elemento principal del mismo es el registro, voluntario, de los individuos y grupos por el que deben inscribirse para poder desarrollar su actividad de forma profesional, legítima y lícita. Esta fórmula contrasta con la aprobada en otros Estados no europeos, como Estados Unidos de América o Canadá que han incorporado desde hace años un modelo que ha ido evolucionando hacia fórmulas más rigurosas de control de la actividad de los lobbies. 
En el caso de las instituciones comunitarias se aprecia un alto grado de participación de las administraciones nacionales y los grupos organizados en el proceso político, debates y foros paralelos que se suceden en el quehacer cotidiano. La ampliación de capacidades y funciones tras Lisboa 2009 ha obligado a la concertación entre las instituciones comunitarias, los gobiernos y demás actores políticos, económicos y sociales involucrados como elementos fundamentales del funcionamiento de la UE en un ejercicio de corresponsabilidad institucional y ciudadana.

Sin embargo el camino en la transparencia no es igual para las instituciones de la UE. El Parlamento y la Comisión llevan la delantera en lo relativo a la transparencia y rendición de cuentas de cara a la ciudadanía. El Consejo Europeo es el órgano más "opaco" en su proceso de toma de decisiones, ya que su enorme red burocrática conexa de más de 10.000 funcionarios es el centro de interés de grupos de lobbies y terceros comprometidos a través de estos.

No existe un marco homogéneo por tanto ni de evaluación ni de sujeción que no sea susceptible de vulneración por parte del ejército lobista permanente en Bruselas, como tampoco hay un marco único en cada uno de los 27. Se trata en general de mecanismos novedosos para regular un oficio que hasta no emerger como parte de la crisis de 2008 no constituyó un problema; muy por el contrario, sigue siendo visto como parte fundamental del ejercicio de la sociedad civil en peticionar al Estado por sus sectoriales intereses.

\section{REFERENCIAS}

Alonso Pelegrín, E. (1995). El Lobby en la Unión Europea: Manual Sobre el buen uso de Bruselas. Madrid: ESIC.

Álvarez, M. y De Montalvo, F. (2014). Los lobbies en el marco de la Unión Europea: una reflexión a propósito de su regulación en España. Teoría y Realidad Constitucional, (33), 353-376. Recuperado de http://dialnet.unirioja.es/descarga/articulo/4724065.pdf

Anastasiadis, S. (2006). Understanding corporate lobbying in its own terms. ICCSR, (42). Recuperado de http:/ / www.nottimgham.ac.uk/business/ICCSR/research.php?action=download\&i $\mathrm{d}=38$

Borchardt, K. (2011). El ABC de la Unión Europea. Luxemburgo: Oficina de Publicaciones de la UE. 
Bouwen, P. (2002). Corporate Lobbying in the European Union: The Logic of Access. Journal of European Public Policy, 3(9), 365-390. Recuperado de http:/ / cadmus.eui.eu/bitstream/handle/1814/318/sps20015.pdf

Castillo, A. (2011). Lobby y comunicación. Sevilla y Zamora: Comunicación Social.

Calduch, R. (2011). Apuntes de la Unión Europea, en Marin, M. (Coord.) Aula Virtual de Relaciones Internacionales, Publicaciones Web. Recuperado de http://pendientedemigracion.ucm.es/info/sdrelint/apuntue.pdf

Comisión Europe. Recuperado de http:/ / ec.europa.eu/civil_service/docs/directors_general/petite_en.pdf

Consejo Europeo (2015). El Consejo Europeo. El órgano estratégico de la Unión Europea, en Documentos y Publicaciones. Bruselas: Consejo Europeo. Recuperado de http:/ / www.consilium.europa.eu/es/ documentspublications/publications/2015/european-council-strategic-body-of-eu/

Correa, R. (2010). Comunicación: lobby y asuntos públicos. Cuaderno 33, 101-110. Recuperado de http:/ / www.scielo.org.ar/pdf/ccedce/n33/n33a09.pdf

El País (2014). Así actúan los lobbies en la UE, en Especial Europa. Recuperado de http:/ / internacional.elpais.com/internacional/2014/04/29/actualidad/1398789625_ 477383.html

Europe Union (2010). The principle of cooperation between the institutions, en EUR Lex, European Union Law. Disponible en:

http:/ / eur-lex.europa.eu/legalcontent/EN/TXT/ ?qid=1453376500757\&uri=URISERV:110125

Francés J. (2013). ¡Qué vienen los lobbies!, en Destino, Barcelona: La Vanguardia. Recuperado de www.lavanguardia.com/libros/20130429/54372866931/entrevistajuan-frances -lobbies.html

Gammelin, C. (2014). La vida en una Ley, en Diario El País de España (mayo 7 de 2014). Recuperado de http:/ / internacional.elpais.com/internacional/2014/05/05/actualidad/1399292152_ 089478.html

García, J. (2008). Modelos de regulación del Lobby en el derecho comparado. Revista Chilena de Derecho, 35(1), 107-134. Recuperado de http:/ / www.scielo.cl/pdf/rchilder/v35n1/art05.pdf 
Guilló, M. y Mancebo-Aracil, J. (2017). Comunicación y participación online: la evolución de los procesos participativos en entornos virtuales. Communication Journal, (8), 413-434. doi: http://dx.doi.org/10.21134/mhcj.v0i8.198

Hernández, J. (2013). Los lobbies financieros. Tentáculos del poder. Madrid: Clave Intelectual.

Hrebenar, R. y Thomas, C. (2008). Understanding interest groups, lobbying an d lobbysts in developing democracies. Jornal of Public Affairs, 8(1), 1-14. Recuperado de http:/ / onlinelibrary.wiley.com/doi/10.1002/pa.v8:1/2/issuetoc

International Transparency (2015). Lobbying in Europe. Hidden Influence, Privileged Acces. París: IT y Co-funded by the Prevention of and Fight against Crime Programme of the European Union. Recuperado de http://www.transparencyinternational.eu/wpcontent/uploads/2015/04/Lobbying_web.pdf

Join Transparency Register Secretariat (2012). Annual Report on the operations of the Transparency Register 2012. Bruselas Secretaries General of the European Parliament and the European Commission. Recuperado de http:/ / ec.europa.eu/transparencyregister/public/staticPage/displayStaticPage.do?1 ocale $=$ es\&reference $=$ ANNUAL_REPORT.

Morata, F. (1995). Influir para decidir: la incidencia de los lobbies en la Unión Europea. Revista de Estudios Políticos, (90), 131. Recuperado de http:// dialnet.unirioja.es/descarga/articulo/27357.pdf

Moriche, J. F. (2012). Puertas giratorias, Madrid: Attac.

Pearson, F. y Rochester, J. (2000). Relaciones Internacionales Situación global en el siglo XXI. Bogotá: Mc Graw-Hill.

Peña, A. (2011). Relaciones públicas y lobby. Gestión para la transparencia. Proyectos de Graduación. Recuperado de http:/ / fido.palermo.edu/servicios_dyc/proyectograduacion/archivos/447.pdf

Peschard, J. (2001). La Cultura Política Democrática. México DF: Instituto Federal Electoral.

Registro de Trasparencia de la UE (2016). Estadísticas, en Portal de datos abiertos de la Unión Europea. Disponible en:

http:/ / ec.europa.eu/transparencyregister/public/homePage.do?redir=false HYPERLINK 
Transparency International (2015). Lobbying in Europe, hidden influence, privileged Access. Recuperado de https://www.transparency.org/whatwedo/publication/lobbying_in_europe

Transparency International (2012). Tratado de la Unión Europea (TUE). Recuperado de http:/ / eur-lex.europa.eu/legalcontent/ES/TXT/PDF/?uri=CELEX:12012M/TXT\&from=ES

Transparency International (2010). Tratado de Funcionamiento de la Unión Europea (TFUE). Recuperado de https://www.boe.es/doue/2010/083/Z00047-00199.pdf

Ure, M. (2018). Engagement estratégico y encuentro conversacional en los medios sociales. Revista De Comunicación, 17(1), 181-196. doi: https:// doi.org/10.26441/RC17.1-2018-A10

Xifra, J. (2005). Los think tank y advocacy tank como actores de la comunicación política. Anàlisi, (32), 73-91. Recuperado de http://www.raco.cat/index.php/analisi/article/viewFile/15173/179893

\section{AUTORA}

\section{Ana Belén Oliver González}

Doctora en Ciencias Políticas y Relaciones Internacionales, Universidad Complutense. Tesis titulada: "Efectos de la comunicación persuasiva de los lobbies en las decisiones de las instituciones comunitarias y países miembros entre 2005 y 2015". Máster universitario en Comunicación Política y Empresarial, Universidad Camilo José Cela. Trabajo Fin de Máster: "Influencia de las agencias Lobby en la Unión Europea. Comunicación persuasiva en las instituciones políticas de Bruselas durante el periodo 1995 al 2010". Grado en Protocolo y Relaciones Institucionales, Universidad Camilo José Cela. Trabajo Fin de Grado: "El protocolo en la ceremonia de investidura de Doctor Honoris Causa". anabeleo@ucm.es 